\title{
Effective reformulations of the truss topology design problem
}

\author{
Michal Kočvara* and Jiří V. Outrata**
}

\begin{abstract}
We present a new formulation of the truss topology problem that results in unique design and unique displacements of the optimal truss. This is reached by adding an upper level to the original optimization problem and formulating the new problem as an MPCC (Mathematical Program with Complementarity Constraints). We derive optimality conditions for this problem and present several techniques for its numerical solution. Finally, we compare two of these techniques on a series of numerical examples.
\end{abstract}

\section{Introduction}

A truss is an assemblage of pin-jointed uniform straight bars. The bars are subjected to only axial tension and compression when the truss is loaded at the joints. With a given load and a given set of joints at which the truss is fixed, the goal of the designer is to find a truss that is as light as possible and satisfies the equilibrium conditions. In the simplest, yet meaningful, approach, the number of the joints (nodes) and their position are kept fixed. The design variables are the bar volumes and the only constraints are the equilibrium equation and an upper bound on the weighted sum of the displacements of loaded nodes, so-called compliance. Recently, this model (or its equivalent reformulations) has been extensively analyzed in the mathematical and engineering literature (see, e.g., $[1,4,12])$.

It is well-known that, when using a small-displacement model, various formulations of the truss topology optimization problem do not have a unique solution. The non-uniqueness may concern both, the bar volumes and the displacements. The nonuniqueness in displacement may be either at the "free" nodes (nodes that, at the optimum, are not connected to the struture by any bar) or at nodes that lie on a straight bar and are otherwise not connected to the rest of the structure. The two cases are demonstrated in Figure 1. On the left-hand side is the initial structure; all bars have

\footnotetext{
* Institute of Applied Mathematics, University of Erlangen, Martensstr. 3, 91058 Erlangen, Germany (kocvara@am. uni-erlangen. de). On leave from the Academy of Sciences of the Czech Republic.

** Institute of Information Theory and Automation, Academy of Sciences of the Czech Republic, Pod vodárenskou věží 4, 18208 Praha 8, Czech Republic (outrata@utia.cas.cz).
} 

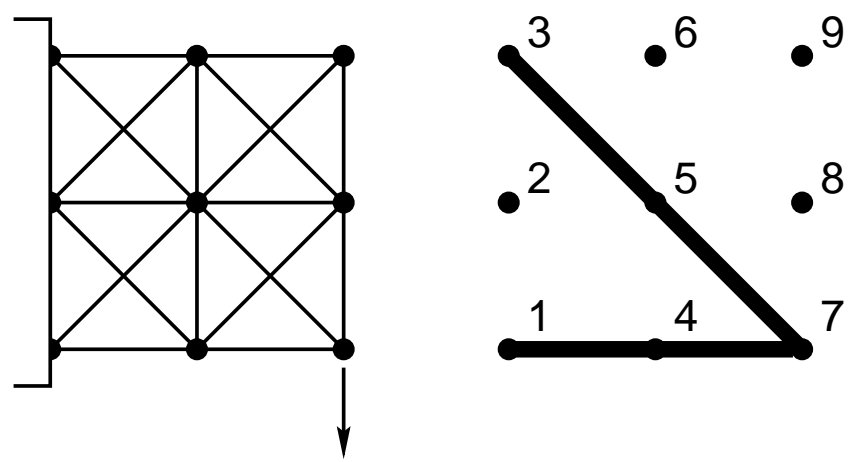

Figure 1: Example non-uniqueness in truss topology design

the same volume. The right-hand side shows the optimal structure. Nodes number 4 , 5, 6, 8 and 9 have non-unique displacements, whereas nodes 6,8,9 represent the first case (free nodes) and nodes 4 and 5 the second case. It is the second case that causes troubles in many generalizations of the truss design problem. For instance, we may face difficulties when we want to add additional constraints on the displacements at certain node (e.g. node 4 in Figure 1); see [13].

The goal of this paper is to give a formulation of the truss topology problem that results in unique displacements and bar volumes of the optimal truss. This is reached by adding an upper level to the original optimization problem and formulating the new problem first as an MPGE (Mathematical Program with a Generalized Equation Constraint) and later as an MPCC (Mathematical Program with Complementarity Constraints). We first give effective formulation of the truss analysis problem (with fixed bar volumes) and later of the truss design problem.

We would like to point out that all MPGE and MPCC problems in this paper are of a special type. They do not work with the usual pair of control and state variables; instead, they only include one, so-called decision variable. An MPGE problem from this class can be written as

$$
\begin{aligned}
& \min _{y} \varphi(y) \\
& \text { s.t. } \quad 0 \in F(y)+Q(y),
\end{aligned}
$$

where $y \in \mathbb{R}^{\ell}, \varphi\left[\mathbb{R}^{\ell} \rightarrow \mathbb{R}\right], F\left[\mathbb{R}^{\ell} \rightarrow \mathbb{R}^{\ell}\right]$ and $Q\left[\mathbb{R}^{\ell} \rightsquigarrow \mathbb{R}^{\ell}\right]$. (With $Q(\cdot)=N_{\mathbb{R}_{+}^{\ell}}(\cdot)$, MPGE becomes MPCC.) That means, we want to identify one specific solution of the generalized equation

$$
0 \in F(y)+Q(y) \text {. }
$$

In the second part of the paper we derive optimality conditions for our problem, while in the third part we present several techniques for its numerical solution. Finally, we compare two of these techniques on a series of numerical examples.

We employ the following notation: For an $[m \times n]$ matrix $A$ and an index set $I \subset\{1,2, \ldots, n\}, A_{I}$ denotes the submatrix of $A$ with columns specified by $I$. Analogously, for a vector $z \in \mathbb{R}^{n}, z_{I}$ denotes the subvector, composed from the components $z_{i}, i \in I$. Furthermore, $\mathbb{B}$ is the unit ball and $\mathcal{R}(H)$ denotes the range of the matrix $H$. 


\section{The truss topology problem and its reformulations}

Consider a truss characterized by the set of nodes, bars and nodal forces. Some of the nodes are assumed to be fixed, that is, some components of the displacement vector are forced to be zero; let us denote by $n$ the number of free displacement components. The nodal positions, forces and displacements are assembled in vectors $x \in \mathbb{R}^{n}, f \in \mathbb{R}^{n}$ and $u \in \mathbb{R}^{n}$, respectively. The bar volumes are denoted by $t_{i}, i=1, \ldots, m$, where $m$ is the number of bars.

\subsection{The truss analysis problem}

Consider first the problem of finding displacements for a given truss, i.e., a given vector $t$. This is a convex quadratic problem of minimizing the potential energy subject to possible unilateral contact constraints:

$$
\min _{u \in \mathbb{R}^{n}} \frac{1}{2} u^{T} A u+f^{T} u
$$

subject to

$$
C u \geq 0 \text {. }
$$

Here $A=A(t) \in \mathbb{R}^{n \times n}$ is the (symmetric and positive semidefinite) stiffness matrix of the truss and $C \in \mathbb{R}^{p \times n}$ contains the unilateral contact information. This problem does not have a unique solution, in general. The stiffness matrix may even have many zero eigenvalues. This is particularly true when $t$ is obtained as a result of topology optimization (see the next section). There is, however, a unique "physical" solution of problem (1). This solution can be identified as a minimizer of a strictly convex continuously differentiable functional $\mathcal{F}(u)$ over all solutions of (1). The construction of $\mathcal{F}(u)$ is explained in Section 5. That means, the physical solution can be found by solving the following MPGE problem:

$$
\min _{u \in \mathbb{R}^{n}} \mathcal{F}(u)
$$

subject to

$$
u \text { solves (1). }
$$

We can further replace the lower-level problem (1) by the corresponding optimality conditions and get an $\mathrm{MPCC}^{1}$ :

$$
\begin{aligned}
& \quad \min _{u \in \mathbb{R}^{n}, \lambda \in \mathbb{R}^{p}} \mathcal{F}(u) \\
& \text { subject to } \\
& \quad A u+f-C^{T} \lambda=0 \\
& \quad 0 \leq C u \quad \perp \quad \lambda \geq 0 .
\end{aligned}
$$

\footnotetext{
${ }^{1}$ Symbol $\perp$ is used for complementarity of the vectors on its left and right-hand side.
} 


\subsection{The truss design problem}

In the traditional formulation of the truss topology problem, one minimizes the compliance (maximizes the stiffness) of the truss subject to equilibrium conditions:

$$
\begin{aligned}
& \min _{t \in \mathbb{R}^{m}, u \in \mathbb{R}^{n}}-f^{T} u \\
& \text { subject to } \\
& \quad\left(\sum_{i=1}^{m} t_{i} A_{i}\right) u+f=0 \\
& \sum_{i=1}^{m} t_{i} \leq 1 \\
& t_{i} \geq 0, \quad i=1, \ldots, m ;
\end{aligned}
$$

here $A_{i} \in \mathbb{R}^{n \times n}$ are symmetric and positive semidefinite element stiffness matrices. It is well-known that this (nonconvex) problem is extremely difficult to solve by standard NLP codes. From the codes recently available on the NEOS ${ }^{2}$ server, only SNOPT can solve bigger than trivial problems.

It was shown by Ben-Tal and Bendsøe [3] that (4) can be equivalently formulated as a convex quadratic problem with quadratic constraints

$$
\begin{aligned}
& \min _{\alpha \in \mathbb{R}, u \in \mathbb{R}^{n}} \alpha-f^{T} u \\
& \text { subject to } \\
& \quad \frac{1}{2} u^{T} A_{i} u-\alpha \leq 0, \quad i=1, \ldots, m .
\end{aligned}
$$

This new formulation can be efficiently solved by interior point methods (see, e.g., [11]). The vector of optimal bar volumes $t$ (part of the optimal solution to (4)), is equal, up to a scaling, to the KKT vector corresponding to inequality constraints in (5). In the following we will ignore this scaling and simply denote the KKT vector by $t$.

Problem (5) does not have a unique solution, in general. As before, the stiffness matrix $\sum_{i=1}^{m} t_{i} A_{i}$ may have (for optimal $t$ ) many zero eigenvalues. This is typical when working with so-called ground structure approach. Here we start with a dense mesh of potential bars; only few of them may be present $\left(t_{i}>0\right)$ at the optimum.

But not only the solution $u$ of (5), also the corresponding KKT vector $t$ is not unique. So, in this case, we would like to identify both, the unique (possible "physical") solution $u$ and the unique KKT vector $t$. Again, this goal can be reached by minimizing a strictly convex continuously differentiable functional $\mathcal{G}(t, u)$ over all solutions (and associated KKT vectors) of (5). In other words, the unique solution of the

\footnotetext{
${ }^{2}$ http://www-neos.mcs.anl.gov/neos/
} 
truss topology problem can be found by solving the following MPGE:

$$
\min _{t \in \mathbb{R}^{m}, u \in \mathbb{R}^{n}} \mathcal{G}(t, u)
$$

subject to

$$
(t, u) \text { solves (5). }
$$

Alternatively, we can formulate the constraint in (6) as

$$
(t, u) \text { solves (4). }
$$

Let us further reformulate this problem as an MPCC. To do that, we write down optimality conditions for (convex) problem (5); the result is a complementarity problem. Then we put these conditions as new constraints in the above problem to get

$$
\min _{t \in \mathbb{R}^{m}, u \in \mathbb{R}^{n}, \alpha \in \mathbb{R}} \mathcal{G}(t, u)
$$

subject to

$$
\begin{aligned}
& \left(\sum_{i=1}^{m} t_{i} A_{i}\right) u+f=0 \\
& \sum_{i=1}^{m} t_{i} \leq 1 \\
& 0 \leq\left(\alpha-\frac{1}{2} u^{T} A_{i} u\right) \quad \perp \quad t_{i} \geq 0, \quad i=1, \ldots, m .
\end{aligned}
$$

\section{Optimality conditions}

In [16], necessary optimality conditions were derived for a class of MPCCs on the basis of the generalized differential calculus of B. Mordukhovich. To derive optimality conditions of this type for the MPCC (7), consider first an abstract problem

$$
\begin{aligned}
& \min \varphi\left(y^{1}, y^{2}\right) \\
& \text { subject to } \\
& \quad H(y)=0 \\
& \quad 0 \leq F(y) \quad \perp \quad y^{2} \geq 0,
\end{aligned}
$$

where $y=\left(y^{1}, y^{2}\right) \in \mathbb{R}^{\ell}, y^{2} \in \mathbb{R}^{\mu}(\mu<\ell), \varphi\left[\mathbb{R}^{\ell} \rightarrow \mathbb{R}\right]$ is locally Lipschitz and $F\left[\mathbb{R}^{\ell} \rightarrow \mathbb{R}^{\mu}\right], H\left[\mathbb{R}^{\ell} \rightarrow \mathbb{R}^{\nu}\right]$ are continuously differentiable. Let us associate with a locally optimal variable $\hat{y}$ in (8) the index sets

$$
\begin{aligned}
L(\hat{y}) & :=\left\{i \in\{1,2, \ldots, \mu\} \mid \hat{y}_{i}^{2}>0\right\} \\
I_{+}(\hat{y}) & :=\left\{i \in\{1,2, \ldots, \mu\} \mid F_{i}(\hat{y})>0\right\} \\
I_{0}(\hat{y}) & :=\left\{i \in\{1,2, \ldots, \mu\} \mid \hat{y}_{i}^{2}=0, F_{i}(\hat{y})=0\right\} .
\end{aligned}
$$

The argument $\hat{y}$ will be dropped whenever these index sets occur as subscripts. 
Theorem 3.1. Consider problem (8) and assume that $\hat{y}=\left(\hat{y}^{1}, \hat{y}^{2}\right)$ is its local solution. Further suppose that $\nabla H(\hat{y})$ is surjective and that the constraint qualification

$$
\left.\begin{array}{l}
{\left[\begin{array}{c}
0 \\
w
\end{array}\right]-(\nabla F(\hat{y}))^{T} v \in \mathcal{R}\left[(\nabla H(\hat{y}))^{T}\right]} \\
w_{L}=0, v_{I_{+}}=0 \text { and for } i \in I_{0}(\hat{y}) \\
\text { either } w_{i} v_{i}=0 \text { or } w_{i}<0 \text { and } v_{i}>0
\end{array}\right\} \quad \text { implies } w=v=0\left(\in \mathbb{R}^{\mu}\right)
$$

holds true. Then there exist multipliers $\hat{w}, \hat{v} \in \mathbb{R}^{\mu}$ and $\hat{\lambda} \in \mathbb{R}^{\nu}$ such that

$$
0 \in \partial \varphi(\hat{y})+\left[\begin{array}{c}
0 \\
w
\end{array}\right]-(\nabla F(\hat{y}))^{T} \hat{v}+(\nabla H(\hat{y}))^{T} \hat{\lambda}
$$

and

$$
\begin{aligned}
& \text { for } i \in L(\hat{y}) \text { one has } \hat{w}_{i}=0, \\
& \text { for } i \in I_{+}(\hat{y}) \text { one has } \hat{v}_{i}=0, \\
& \text { for } i \in I_{0}(\hat{y}) \text { one has either } \hat{v}_{i} \hat{w}_{i}=0 \text { or } \hat{w}_{i}<0 \text { and } \hat{v}_{i}>0 \text {. }
\end{aligned}
$$

Proof. The statement follows directly from [16, Thm.3.2] by putting $x=y^{1}, y=y^{2}$ and $\Omega=\left\{y \in \mathbb{R}^{\ell} \mid H(y)=0\right\}$. To be able to express the normal cone $N_{\Omega}(\hat{y})$ in terms of $H$, we assume the surjectivity of $\nabla H(\hat{y})$.

Problem (7) can be written down in the form of (8): we just put $\ell=n+1+m$, $\mu=m, \nu=n+1, y^{1}=(u, \alpha), y^{2}=t, \varphi(y)=\mathcal{G}(t, u)$,

$$
F(y)=\left[\begin{array}{c}
\alpha-\frac{1}{2}\left\langle u, A_{1} u\right\rangle \\
\vdots \\
\alpha-\frac{1}{2}\left\langle u, A_{m} u\right\rangle
\end{array}\right]
$$

and

$$
H(y)=\left[\begin{array}{c}
\left(\sum_{i=1}^{m} t_{i} A_{i}\right)_{m} u+f \\
-1+\sum_{i=1}^{m} t_{i}
\end{array}\right] .
$$

This is legal because in problem (7) the inequality $\sum_{i=1}^{m} t_{i} \leq 1$ can be replaced by the equality $\sum_{i=1}^{m} t_{i}=1$.

Necessary optimality conditions for (7) can now be stated as follows.

Theorem 3.2. Let $\hat{y}=(\hat{u}, \hat{\alpha}, \hat{t})$ be a local solution to $(7)$ and define the $[(n+1) \times m]$ matrix

$$
D(\hat{u}, \hat{\alpha}):=\left[\begin{array}{ccc}
-A_{1} \hat{u} & \cdots & -A_{m} \hat{u} \\
1 & \cdots & 1
\end{array}\right] .
$$

Assume that the matrices $(D(\hat{u}, \hat{\alpha}))_{L \cup I_{0}}$ and

$$
\left[\begin{array}{cc}
\sum_{i=1}^{m} \hat{t}_{i} A_{i} & 0 \\
0_{1 \times n} & 0 \\
\left(A_{1} \hat{u}\right)^{T} & 1 \\
\vdots & \vdots \\
\left(A_{m} \hat{u}\right)^{T} & 1
\end{array}\right]
$$


have the full column rank. Then there exist multiplies $\hat{\lambda} \in \mathbb{R}^{n}$, $\hat{\alpha} \in \mathbb{R}, \hat{w}, \hat{v} \in \mathbb{R}^{m}$ such that

$$
\begin{aligned}
0 & =\nabla_{v} \mathcal{G}(\hat{t}, \hat{u})-\left(\sum_{i \in L \cup I_{0}} \hat{v}_{i} A_{i}\right) \hat{u}+\left(\sum_{i=1}^{m} \hat{t}_{i} A_{i}\right) \hat{\lambda} \\
0 & =\sum_{i \in L \cup I_{0}} \hat{v}_{i} \\
0 & =\nabla_{t_{i}} \mathcal{G}(\hat{t}, \hat{u})+\hat{w}_{i}+\left\langle A_{i} \hat{u}, \hat{\lambda}\right\rangle+\hat{\alpha}, \quad i=1,2, \ldots, m .
\end{aligned}
$$

Moreover, vectors $\hat{w}, \hat{v}$ fulfill the relations from Theorem 3.1.

Proof. The statement follows directly from Theorem 3.1, because the injectivity of $(D(\hat{u}, \hat{\alpha}))_{L \cup I_{0}}$ ensures (together with $\left.v_{I_{+}}=0\right)$ the satisfaction of the constraint qualification and the injectivity of (10) amounts to the surjectivity of $\nabla H(\hat{y})$.

In the next illustrative example the objective $\mathcal{G}$ depends only on the variable $u$.

Example 1. Consider the following MPCC:

$$
\begin{aligned}
& \min _{u \in \mathbb{R}^{2}, t \in \mathbb{R}^{2}, \alpha \in \mathbb{R}^{1}} \frac{1}{2}\left(u_{2}+0.3\right)^{2} \\
& \text { subject to } \\
& \quad\left(2 t_{1}+t_{2}\right) u_{1}-t_{2} u_{2}=1 \\
& \quad-t_{2} u_{1}+t_{2} u_{2}=0 \\
& \quad t_{1}+t_{2} \leq 1 \\
& \quad 0 \leq\left(\alpha-u_{1}^{2}\right) \quad \perp \quad t_{1} \geq 0 \\
& \quad 0 \leq\left(\alpha-\frac{1}{2}\left(u_{1}-u_{2}\right)^{2}\right) \quad \perp \quad t_{2} \geq 0 .
\end{aligned}
$$

The solution is

$$
\hat{u}=\left(\frac{1}{2},-\frac{1}{2}+\sqrt{\frac{1}{2}}\right), \quad \hat{t}=(1,0), \quad \hat{\alpha}=\frac{1}{4} .
$$

One easily computes $A_{1}=\left[\begin{array}{ll}2 & 0 \\ 0 & 0\end{array}\right], A_{2}=\left[\begin{array}{cc}1 & -1 \\ -1 & 1\end{array}\right]$ and realizes that $L=\{1\}$ and $I_{0}=\{2\}$. The gradient of the objective amounts to $\hat{u}_{2}+0.3=-0.2+\sqrt{\frac{1}{2}}$ and

$$
D(\hat{u}, \hat{\alpha})=\left[\begin{array}{cc}
-1 & -1+\sqrt{\frac{1}{2}} \\
0 & 1-\sqrt{\frac{1}{2}} \\
1 & 1
\end{array}\right] .
$$


Hence the constraint qualification is fulfilled and also the injectivity of the respective matrix (10) can easily be verified. The linear system (11) attains the form

$$
\left[\begin{array}{c}
0 \\
0.2-\sqrt{\frac{1}{2}} \\
0 \\
0 \\
0
\end{array}\right]=\left[\begin{array}{cccccc}
0 & 1 & 1-\sqrt{\frac{1}{2}} & 0 & 2 & 0 \\
0 & 0 & -1+\sqrt{\frac{1}{2}} & 0 & 0 & 0 \\
0 & -1 & -1 & 0 & 0 & 0 \\
0 & 0 & 0 & 1 & 1 & 0 \\
1 & 0 & 0 & 1 & 1-\sqrt{\frac{1}{2}} & -1+\sqrt{\frac{1}{2}}
\end{array}\right]\left[\begin{array}{c}
w_{2} \\
v_{1} \\
v_{2} \\
\alpha \\
\lambda_{1} \\
\lambda_{2}
\end{array}\right]
$$

We can thus set, e.g., $\hat{w}_{1}=0, \hat{w}_{2}=0, \hat{v}_{1}=-1.73, \hat{v}_{2}=1.73, \hat{\alpha}=-0.606$, $\hat{\lambda}_{1}=0.606$, and $\hat{\lambda}_{2}=-1.462$. Observe that all additional conditions imposed on the multipliers $\hat{w}, \hat{v}$ are fulfilled.

\section{Solution approaches}

In this section we present three approaches to the numerical solution of an optimization problem of the type

$$
\begin{aligned}
& \min _{y} \varphi(y) \\
& \text { s.t. } \quad 0 \in F(y)+Q(y) \text {, }
\end{aligned}
$$

where $y \in \mathbb{R}^{\ell}, \varphi\left[\mathbb{R}^{\ell} \rightarrow \mathbb{R}\right]$ is assumed to be continuously differentiable, $F\left[\mathbb{R}^{\ell} \rightarrow \mathbb{R}^{\ell}\right]$ is continuously differentiable and $Q\left[\mathbb{R}^{\ell} \rightsquigarrow \mathbb{R}^{\ell}\right]$ has a closed graph. That means, we want to identify one, possibly unique, solution of the generalized equation

$$
0 \in F(y)+Q(y) .
$$

With $Q(\cdot)=N_{\mathbb{R}_{+}^{e}}(\cdot)$, problem (12) becomes MPCC mentioned in the Introduction. Two from the methods proposed in the sequel are tailored to this particular form of $\mathrm{Q}$.

Needless to say that our reformulation of truss topology problem is of type (12).

\subsection{Exact penalty}

Let us first recall from [17] that a multifunction $\Phi\left[\mathbb{R}^{s} \rightsquigarrow \mathbb{R}^{p}\right]$ with a closed graph is calm at $(\bar{v}, \bar{w}) \in \mathrm{Gph} \Phi$, provided there exist neighborhoods $\mathcal{V}$ of $\bar{v}, \mathcal{W}$ of $\bar{w}$ and a scalar $L>0$ such that

$$
\Phi(v) \cap \mathcal{W} \subset \Phi(\bar{v})+L\|v-\bar{v}\| \mathbb{B} \quad \text { for all } v \in \mathcal{V} .
$$

Now assume that the map

$$
\left(\xi_{1}, \xi_{2}\right) \mapsto\left\{y \in \mathbb{R}^{\ell} \mid\left(\begin{array}{c}
\xi_{1}+y \\
\xi_{2}-F(y)
\end{array}\right) \in \operatorname{Gph} Q\right\}
$$


is calm at $(0,0, \hat{y})$, where $\hat{y}$ is a local solution of (12). Then $\hat{y}$ is a local minimizer of the penalized objective

$$
\varphi(y)+R \operatorname{dist}_{\mathrm{Gph} Q}\left(\begin{array}{c}
y \\
-F(y)
\end{array}\right)
$$

provided $R$ is sufficiently large. This approach has, unfortunately, a big drawback; the penalty term is highly nonconvex and we need to find its global minimum (zero).

\subsection{Minimizing over solution set}

Assume that $F(\cdot)=\nabla f(\cdot)$ and $Q(\cdot)=N_{K}(\cdot)$, where $f\left[\mathbb{R}^{\ell} \rightarrow \mathbb{R}\right]$ is convex and continuously differentiable and $K \subset \mathbb{R}^{\ell}$ is closed and convex. Then (13) corresponds to the convex optimization problem

$$
\begin{aligned}
& \min _{y \in \mathbb{R}^{\ell}} f(y) \\
& \text { subject to } \\
& \quad y \in K .
\end{aligned}
$$

The solution set $\mathcal{S}$ of this problem is convex and can be fully characterized by the problem data and one arbitrary solution of (14). Solving (12) then amounts to minimizing $\varphi$ on this solution set ${ }^{3}$. The characterization of $\mathcal{S}$ is based on the following proposition that directly follows from the definition of a solution.

Proposition 4.1. Let $y^{*}$ be a solution of (14). Then $\bar{y}$ is a solution of (14) if and only if

$$
\begin{aligned}
\bar{y} & \in K \\
f(\bar{y}) & =f\left(y^{*}\right) .
\end{aligned}
$$

When $f(y)=\frac{1}{2} y^{T} M y+g^{T} y$ with a positive semidefinite $M$, condition (16) amounts to

$$
M\left(\bar{y}-y^{*}\right)=0, \quad g^{T}\left(\bar{y}-y^{*}\right)=0 .
$$

Applying this to our truss problems, we get the following equivalent formulations of (2) and (6).

Corollary 4.2. Let $u^{*}$ be a solution of (1). Then $\widehat{u}$ is the solution of the truss analysis problem (2) if and only if it solves the following problem

$$
\begin{aligned}
& \min _{u \in \mathbb{R}^{n}} \mathcal{F}(u) \\
& \text { subject to } \\
& \quad C u \geq 0 \\
& A\left(u-u^{*}\right)=0 \\
& f^{T}\left(u-u^{*}\right)=0 .
\end{aligned}
$$

\footnotetext{
${ }^{3}$ This fact was pointed out by J.-S. Pang.
} 
Application of this approach to the truss design problem (6) is not so straightforward, because (4) is not a convex problem and Proposition 4.1 does not apply. On the other hand, problem (5) does not contain $t$ as a (primal) variable and can thus be only used in case when $\mathcal{G}$ does not depends on $t$.

Corollary 4.3. Assume that $\mathcal{G}$ only depends on $u$. Let $\left(\alpha^{*}, u^{*}\right)$ be a solution of (5). Then $\widehat{u}$ is the solution of the truss design problem (6) if and only if it solves the following problem

$$
\begin{aligned}
& \min _{u \in \mathbb{R}^{n}} \mathcal{G}(u) \\
& \text { subject to } \\
& \quad \frac{1}{2} u^{T} A_{i} u-\alpha \leq 0, \quad i=1, \ldots, m . \\
& \quad \alpha-\alpha^{*}=0 \\
& \quad f^{T}\left(u-u^{*}\right)=0 .
\end{aligned}
$$

Note that both problems (17) and (18) are convex mathematical programs. In the last section we will present results of numerical examples solved by this approach. We will see that problems (17) and (18) can be solved almost equally efficiently as the underlying problems (1) and (5).

\subsection{NLP reformulation}

This technique has been recently proposed by several authors [2, 8, 9]. Assume that $Q(\cdot)=N_{\mathbb{R}_{+}^{\ell}}(\cdot)$; problem (12) is then an MPCC. The technique consists in reformulation of this MPCC problem as an NLP one. In particular, one replaces the complementarity constraint of type

$$
0 \leq z_{1} \perp z_{2} \geq 0
$$

by inequalities

$$
z_{1} \geq 0, \quad z_{2} \geq 0, \quad z_{2}^{T} z_{1} \leq 0 .
$$

The new problem, although it does not satisfy standard constraint qualification, can often be solved by standard NLP methods, both of SQP and interior-point type [2, 8, 5]. Fletcher and Leyffer [8] report on results of three SQP codes, namely their own code FilterSQP, KNITRO [6] and SNOPT [10], on a collection of 137 MPCC problems. Two of these codes fail to find a solution only in 5 and 7 cases, respectively. Because the MPCC problem does not satisfy usual NLP constraint qualifications, it may happen that the quadratic programming subproblem in SQP is infeasible. The robustness of SQP based codes is then increased by various techniques, like the elastic mode in SNOPT [10]. 
In our case we write the truss analysis problem (3) as

$$
\begin{aligned}
& \min _{u \in \mathbb{R}^{n}, \lambda \in \mathbb{R}^{p}} \mathcal{F}(u) \\
& \text { subject to } \\
& A u+f-C^{T} \lambda=0 \\
& C u \geq 0 \\
& \lambda \geq 0 \\
& \lambda^{T} C u \leq 0 .
\end{aligned}
$$

The NLP formulation of the truss design problem (7) reads as follows:

$$
\min _{t \in \mathbb{R}^{m}, u \in \mathbb{R}^{n}, \alpha \in \mathbb{R}} \mathcal{G}(t, u)
$$

subject to

$$
\begin{aligned}
\left(\sum_{i=1}^{m} t_{i} A_{i}\right) u+f & =0 \\
\sum_{i=1}^{m} t_{i} & \leq 1 \\
\alpha-\frac{1}{2} u^{T} A_{i} u & \geq 0, \quad i=1, \ldots, m \\
t_{i} & \geq 0, \quad i=1, \ldots, m \\
\sum_{i=1}^{m} t_{i}\left(\alpha-\frac{1}{2} u^{T} A_{i} u\right) & \leq 0 .
\end{aligned}
$$

Let us stress that this formulation allows us to solve the general truss design problem (7), without the restrictive assumption of Corollary 4.3.

\section{Choice of $\mathcal{F}$ and $\mathcal{G}$}

In this section we would like to derive functions $\mathcal{F}$ and $\mathcal{G}$ that give a unique and possibly physical solution in MPCCs (3) and (7). The uniqueness can be easily guaranteed by a proper choice of $\mathcal{F}$ and $\mathcal{G}$. What do we mean by a "physical solution"?

Let us start with the truss analysis problem (3). Consider again the $3 \times 3$ example from Figure 1. In the analysis problem, we do not have to take care of the free nodes $6,8,9$, as they can be eliminated from the system. We do have to take care of nodes 4 and 5 , lying on straight bars and having no connection to the rest of the structure. We call displacement of such a node physical if it is a linear combination of the displacements at the end nodes of the bars (nodes 1 and 7 for node 4, nodes 3 and 7 for node 5); that means, straight bars (like 1-4-7 and 3-5-7) remain straight after deformation.

The fact that the displacements may not be unique in the original analysis problem (1) follows from the linear model of trusses. In this model, the strain in a bar is 
computed as a relative prolongation in the direction of the bar axis $x$ before deformation. Consequently, a bar deformed only along its own axis (Fig. 2(a)) gets the same value of strain as a bar with higher deflection that is additionally "rotated" (Fig. 2(b)), even though the true strain should be higher in the second case.

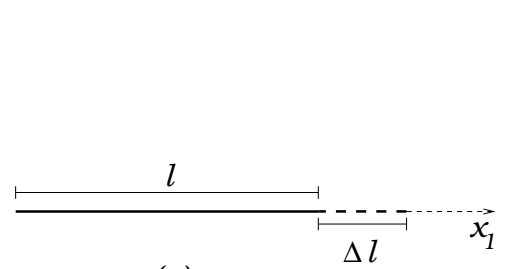

(a)

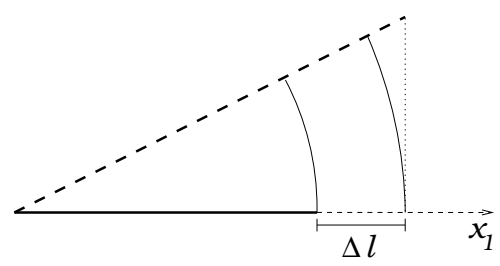

(b)

Figure 2: A bar deformed along its axis (a) and a bar deformed and rotated (b)

Consider a general bar of length $\ell$ in the $2 \mathrm{D}$ space, as shown in Fig. 3. The displace-

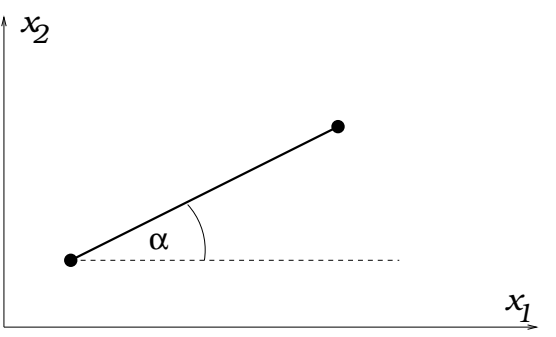

Figure 3: A general bar in the 2-D space

ments are assumed to be linear between the two end-points; let $u=\left(u_{1}, u_{2}, u_{3}, u_{4}\right)$ denote the vector of nodal displacements. The large-deflection strain-displacement relation for this bar is given by

$$
\epsilon=\frac{1}{\ell} \gamma^{T} u+\frac{1}{2}\left(\frac{1}{\ell} \gamma^{T} u\right)^{2}+\frac{1}{2}\left(\frac{1}{\ell} \delta^{T} u\right)^{2}
$$

with

$$
\gamma=\left(\begin{array}{r}
-\cos \alpha \\
-\sin \alpha \\
\cos \alpha \\
\sin \alpha
\end{array}\right), \quad \delta=\left(\begin{array}{r}
\sin \alpha \\
-\cos \alpha \\
-\sin \alpha \\
\cos \alpha
\end{array}\right)
$$

In the linear framework, the deflections are assumed to be small so that the quadratic terms in the strain-displacement relation can be neglected. This has the side-effect of non-unique displacements. To make the displacements of nodes like 4 and 5 in Figure 1 unique and in accordance with the nonlinear model, we want to minimize the component of their displacements that is perpendicular to the bar axis. That means, we 
want to minimize the last term in (21) (which in the nonlinear formulation enters the potential energy that is to be minimized).

Hence, with the stiffness matrix of the truss given by

$$
A(t)=\sum_{i=1}^{m} t_{i} A_{i}, \quad A_{i}=\frac{E_{i}}{\ell_{i}^{2}} \gamma_{i} \gamma_{i}^{T}
$$

we introduce the function $\mathcal{F}$ as

$$
\mathcal{F}(u)=u^{T} \Phi u, \quad \Phi=\sum_{i=1}^{m} \delta_{i} \delta_{i}^{T},
$$

where $\gamma_{i}, \delta_{i}, i=1, \ldots, m$, are vectors from (22) evaluated for particular bars. Matrix $\Phi$ is obviously symmetric and positive definite.

The situation is more complicated in the truss design problem (7). Let us first assume that we are only interested in the uniqueness of $u$ and do not care about possible non-uniqueness of $t$. Hence we may assume that $\mathcal{G}$ is only a function of $u$. The mentioned complication is due to the fact that the formulations (4) and (5) are actually not fully equivalent. Although they give the same optimal value of the compliance, the solution set in (4) is bigger than in (5). Let us demonstrate it again on the example from Figure 1. When we choose $\mathcal{G}$ as $\mathcal{F}$ in (23) and solve the MPCC problem (7), we get the solution shown in Figure 4, left. Here we also depict bars that, at the optimum, have $t_{i}=0$ and are therefore not present in the optimal structure. This solution is certainly not physical; neither of the bars 1-4-7 and 3-5-7 is straight after deformation (for numbering we refer to Figure 1). A physical solution is presented in Figure 4, right. However, although the physical solution has the same value of compliance as the
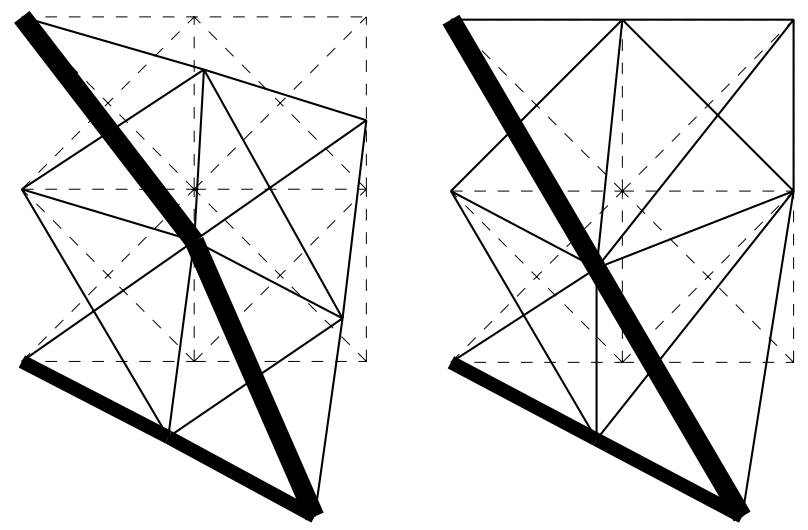

Figure 4: Non-physical (left) and physical (right) solution of the $3 \times 3$ truss design problem. Also bars with zero volumes are presented.

"non-physical" one, and although it is feasible in problem (4), it is not feasible in (5). 
(For instance, bar 7-8 severely violates the quadratic constraint.) This is because, unlike in (4), the constraints in (5) are relevant also for bars that, at the optimum, are not present in the structure at all.

Therefore, if we use as a basis for our MPCC formulation problem (7), we can never reach the physical solution in this example, whatever the choice of $\mathcal{G}$ is. In this light, it seems that the best choice of $\mathcal{G}$ is the same as in (23), because it results in unique displacements that are possibly closest to the physical solution. This was also our choice in the following test examples. If one wants to find a physical solution of the truss optimization problem and does not care about the non-uniqueness of $t$, one has to proceed in a different way: first to solve problem (5) by any method to get an optimal truss characterized by a vector $t^{*}$. Then, to solve the analysis problem (3) with this truss, which gives unique and physical displacements.

Now, as we mentioned above, the optimal vector $t$ may also be non-unique. This is demonstrated in Figure 5: it again shows a $3 \times 3$ truss, this time with all nodes connected by potential bars. Both trusses presented in this figure and all their convex combinations are optimal solutions of problem (4). The criterium for selecting one of these solutions (i.e., function $\mathcal{G}(t, u)$ ) depends on user's preferences. We propose to select a solution vector $t$ with the least number of active bars (bars with nonzero volume). This can be simulated, for instance, by minimizing the function

$$
\mathcal{G}(t, u)=u^{T} \Phi u+\sum_{i=1}^{m} t_{i}^{2}
$$

with $\Phi$ from (23).
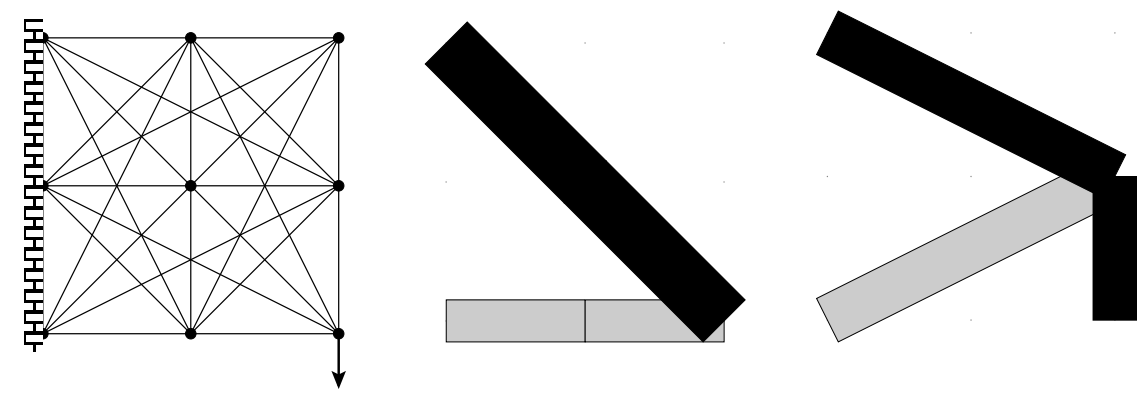

Figure 5: Two solutions of the $3 \times 3$ truss design problem with all nodes connected.

\section{Examples}

We present results of numerical computations using the solution set approach (Section 4.2) and the NLP reformulation (Section 4.3). 


\subsection{The truss analysis problem}

We generated three examples of the truss analysis problem of increasing dimension. Figure 6 shows one such truss-it is the result of the truss optimization problem and we can see that many bars have zero volume. Also, there are many nodes lying on straight bars, a source of possible nonuniqueness. The problems were solved using for-

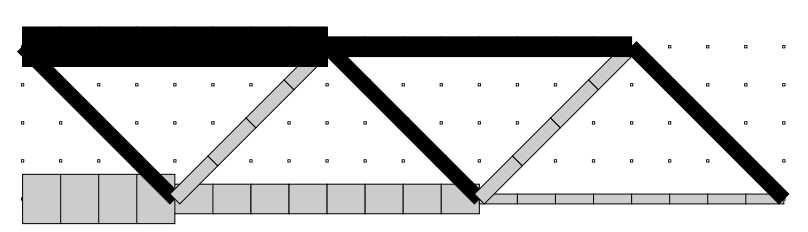

Figure 6: Problem tr21x5. The truss is fixed at the left-hand side nodes and subjected to a vertical load at the right-lower node.

mulations (17) and (19) with function $\mathcal{F}$ chosen as in (23). The resulting optimization problems were solved by NLP codes available on the NEOS server.

Table 1: Results for the "solution set" reformulation (17) of the truss analysis problem. Given are the numbers of iterations for each code. "F" means that the code stopped with a failure message. " $F(3)$ " denotes failure close to the optimal point, with 3 digits of accuracy.

\begin{tabular}{l|rr|rrrrr}
\hline problem & var & constr & LOQO & Filter & SNOPT & MINOS & PENNON \\
\hline tr_11x3 & 60 & 62 & 26 & 4 & $\mathrm{~F}$ & 39 & 9 \\
$\operatorname{tr} 21 \times 5$ & 200 & 202 & 26 & $\mathrm{~F}$ & 100 & 92 & 10 \\
$\operatorname{tr} \_41 \times 9$ & 720 & 722 & $\mathrm{~F}(3)$ & $\mathrm{F}$ & $\mathrm{F}(4)$ & $\mathrm{F}$ & 73 \\
\hline
\end{tabular}

Table 2: NLP reformulation (19) of the truss analysis problem.

\begin{tabular}{l|rr|rrrrr}
\hline problem & var & constr & LOQO & Filter & SNOPT & MINOS & PENNON \\
\hline tr_11x3 & 47 & 34 & 33 & 10 & 63 & 53 & 13 \\
$\operatorname{tr} 21 \times 5$ & 103 & 72 & 34 & 9 & 137 & 123 & 23 \\
tr_41x9 & 569 & 534 & $\mathrm{~F}$ & $\mathrm{~F}$ & $\mathrm{~F}$ & $\mathrm{~F}$ & $\mathrm{~F}$ \\
\hline
\end{tabular}

Table 1 shows the results of the solution set reformulation for the most successful NEOS codes, namely, LOQO [18], FilterSQP [7], SNOPT [10], MINOS [15], and of our code PENNON [14]. Table 2 presents the results of the NLP reformulation for the same codes.

We must remark that "one iteration" has different meaning in different codes, and thus the table basically presents the ability of the code to solve the particular problem. 


\subsection{The truss design problem}

In order to compare the two solution approaches, let us first assume that $\mathcal{G}$ is only a function of $u$. The difference between the two approaches is more dramatic than in the truss analysis problem. On the one hand, (18) is a convex problem that is similar to (5). We know that (5) can be solved very efficiently by interior-point and penalty methods and may expect the same in case of (18). On the other hand, the problem (20) resembles problem (4). As (4) was a difficult NLP problem, we cannot expect (20) to be any simpler and this is clearly seen from our numerical results. It turned out that the examples from the previous section are all too big for the NLP approach, so we generated several smaller ones. These new examples are based on the $3 \times 3$ truss from Figure 1 but include an additional constraint on the horizontal displacement of node 7; it should be nonnegative. Figure 7 shows an optimal $3 \times 3$ truss with this additional constraint, before and after deformation.
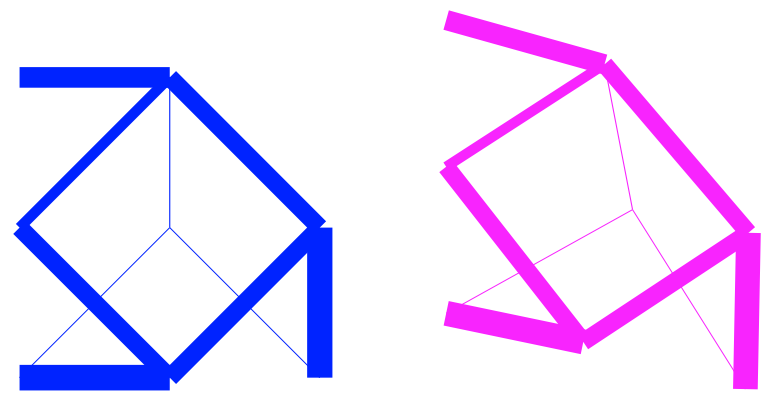

Figure 7: Problem tro 3 x 3 ; optimal solution before and after deformation.

We first solved the new examples (plus the old ones) by the solution set approach, using again the NEOS solvers. Table 3 reports the results-number of iterations needed by each solver. To get a vague idea how do the codes compare with respect to CPU time, the last two rows of Table 3 present CPU times in seconds for the two largest problems. The times for the first four codes were obtained using the NEOS benchmark solver and are thus comparable. The times for PENNON refer to Pentium III $1000 \mathrm{MHz}$ PC running Linux.

The only code capable to solve NLP reformulations of the MPCC (7) was SNOPT, all the other codes failed (MINOS could solve the smallest example in 368 iterations). Table 4 presents the results.

Finally, we present results of the general formulation of the physically meaningful truss design problem, i.e., with $\mathcal{G}$ being a function of $u$ and $t$. Consider a series of examples of increasing dimension, based on the $3 \times 3$ truss with all nodes connected by potential bars; see Figure 5. We take the particular form of $\mathcal{G}$ as defined in (24). As these problems cannot be solved by the solution set approach, we only show results for the NLP reformulation (7). Again, these problems are very difficult to solve, as expected. Table 5 shows results for the successful NEOS solvers. 
Table 3: "Solution set" reformulation (18) of the truss design problem. The last row contains CPU times in seconds.

\begin{tabular}{l|rr|rrrrr}
\hline problem & var & constr & LOQO & Filter & SNOPT & KNITRO & PENNON \\
\hline tro_3x3 & 12 & 20 & 22 & 10 & 36 & 54 & 13 \\
tro_4x4 & 24 & 41 & 21 & 14 & $\mathrm{~F}$ & 99 & 18 \\
tro_5x5 & 40 & 70 & 26 & 17 & 200 & 298 & 18 \\
tro_6x2 & 20 & 27 & 35 & 16 & 139 & 59 & 35 \\
tro_11x3 & 60 & 92 & 55 & 16 & 566 & 188 & 50 \\
tro_21x5 & 200 & 342 & 51 & 24 & 6526 & 830 & 79 \\
tro_41x9 & 720 & 1322 & 134 & $\mathrm{~F}$ & 63416 & $\mathrm{~F}$ & 78 \\
\hline tro_21x5 & 200 & 342 & 1.6 & 4.6 & 13.6 & 22.4 & 1.9 \\
tro_41x9 & 720 & 1322 & 24.3 & n.a. & 419.7 & n.a. & 8.8 \\
\hline
\end{tabular}

Table 4: NLP reformulation (20) of the truss design problem.

\begin{tabular}{l|rr|r}
\hline problem & var & constr & SNOPT \\
\hline tro_3x3 & 31 & 32 & 259 \\
tro_4x4 & 64 & 65 & 593 \\
tro_5x5 & 109 & 110 & 1122 \\
tro_6x2 & 46 & 47 & 763 \\
tro_11x3 & 151 & 152 & $\mathrm{~F}$ \\
\hline
\end{tabular}

All problems from this section are available in AMPL format on the Web page http: //www2 am. uni-erlangen. de/ kocvara/mpec/.

\section{Acknowledgment}

This research was supported by BMBF project 03ZOM3ER and by grant No. 201/00/0080 of the Grant Agency of the Czech Republic.

Table 5: NLP reformulation (20) of the truss design problem.

\begin{tabular}{l|rr|rrr}
\hline problem & var & constr & SNOPT & KNITRO & MINOS \\
\hline tro_3x3f & 40 & 39 & 31 & 623 & 334 \\
tro_4x4f & 111 & 109 & 49 & $\mathrm{~F}$ & 1588 \\
tro_5x5f & 241 & 238 & 4755 & $\mathrm{~F}$ & $\mathrm{~F}$ \\
\hline
\end{tabular}




\section{References}

[1] W. Achtziger, A. Ben-Tal, M. Bendsøe, and J. Zowe. Equivalent displacement based formulations for maximum strength truss topology design. IMPACT of Computing in Science and Engineering, 4:315-345, 1992.

[2] M. Anitescu. On solving mathematical programs with complementarity constraints as nonlinear programs. Preprint ANL/MCS-P864-1200, Argonne National Laboratory, Argonne, IL, 2000.

[3] A. Ben-Tal and M. Bendsøe. A new iterative method for optimal truss topology design. SIAM J. Optimization, 3:322-358, 1993.

[4] M. Bendsøe and O. Sigmund. Topology Optimization. Theory, Methods and Applications. Springer-Verlag, Heidelberg, 2002.

[5] H. Benson, D. Shanno, and R. Vanderbei. Interior point methods for nonconvex nonlinear programming: Complementarity comstraints. Technical Report ORFE-02-02, Operations Research and Financial Engineering, Princeton University, September 2002.

[6] R. Byrd, M. E. Hribar, and J. Nocedal. An interior point method for large scale nonlinear programming. SIAM J. Optimization, 9(4):877-900, 1999.

[7] R. Fletcher and S. Leyffer. Nonlinear programming without a penalty function. Mathematical Programming, 91:239-269, 2002.

[8] R. Fletcher and S. Leyffer. Numerical experience with solving MPECs as NLPs. Report NA $\backslash 210$, University of Dundee, 2002.

[9] R. Fletcher, S. Leyffer, D. Ralph, and S. Scholtes. Local convergence of SQP methods for mathematical programs with equilibrium constraints. Report $\mathrm{NA} \backslash 209$, University of Dundee, 2002.

[10] P. E. Gill, W. Murray, and M. A. Sounders. SNOPT: an SQP algorithm for largescale constrained optimization. SIAM J. Optimization, 12:979-1006, 2002.

[11] F. Jarre, M. Kočvara, and J. Zowe. Interior point methods for mechanical design problems. SIAM J. Optimization, 8(4):1084-1107, 1998.

[12] M. Kočvara, M. Zibulevsky, and J. Zowe. Mechanical design problems with unilateral contact. M2AN Mathematical Modelling and Numerical Analysis, 32:255282, 1998.

[13] M. Kočvara. On the modelling and solving of the truss design problem with global stability constraints. Struct. Multidisc. Optimization, 23(3):189-203, 2000.

[14] M. Kočvara and M. Stingl. PENNON-a code for convex nonlinear and semidefinite programming. Optimization Methods and Software, 2003. In print. 
[15] B. A. Murtagh and M. A. Saunders. MINOS 5.5 user's guide. Report SOL 8320R, Dept of Operations Research, Stanford University, 1998.

[16] J. Outrata. On mathematical programs with complementarity constraints. Optimization Methods and Software, 14:117-137, 2000.

[17] R. T. Rockafellar and R. J-B. Wets. Variational Analysis. Springer, BerlinHeidelberg, 1998.

[18] R. J. Vanderbei and D. F. Shanno. An interior point algorithm for nonconvex nonlinear programming. Computational Optimization and Applications, 13:231252, 1999. 